\title{
Optical spectroscopy of IRAS 02091+6333
}

\author{
S. Kimeswenger ${ }^{1}$, A. Bacher ${ }^{1}$, M. Emprechtinger ${ }^{1}$, G. E. Grömer ${ }^{1}$, W. Kapferer ${ }^{1}$, W. Kausch ${ }^{1}$, \\ M. G. Kitzbichler ${ }^{1}$, M. F. M. Lechner ${ }^{1}$, C. Lederle ${ }^{1}$, K. Uytterhoeven ${ }^{2,3}$, and A. A. Zijlstra ${ }^{4}$ \\ 1 Institut für Astrophysik der Leopold-Franzens-Universität Innsbruck, Technikerstr. 25, 6020 Innsbruck, Austria \\ 2 Instituut voor Sterrenkunde, Katholieke Universiteit Leuven, Celestijnenlaan 200 B, 3001 Leuven, Belgium \\ ${ }^{3}$ Mercator Telescope, Calle Alvarez de Abreu 70, 38700 Santa Cruz de La Palma, Spain \\ 4 Astrophysics Group, Department of Physics, UMIST, PO Box 88, Manchester M60 1QD, UK
}

Received 22 April 2003 / Accepted 16 June 2003

\begin{abstract}
We present a detailed spectroscopic investigation, spanning four winters, of the asymptotic giant branch (AGB) star IRAS 02091+6333. Zijlstra \& Weinberger (2002) found a giant wall of dust around this star and modelled this unique phenomenon. However their work suffered from the quality of the optical investigations of the central object. Our spectroscopic investigation allowed us to define the spectral type and the interstellar foreground extinction more precisely. Accurate multi band photometry was carried out. This provides us with the possibility to derive the physical parameters of the system. The measurements presented here suggest a weak irregular photometric variability of the target, while there is no evidence of a spectroscopic variability over the last four years.
\end{abstract}

Key words. stars: AGB and post-AGB - stars: individual: IRAS 02091+6333 = GSC 04041-01743

\section{Introduction}

When Zijlstra \& Weinberger (2002) discovered a massive dust shell around the AGB star IRAS 02091+6333 they could use only a single quick look spectrum and the original TYCHO $B_{\mathrm{T}}$ and $V_{\mathrm{T}}$ magnitudes (Perryman et al. 1997) to estimate the spectral type and the interstellar extinction towards the target. The spectroscopic investigation was lacking comparison spectra obtained with the same instrument setup. Furthermore the TYCHO instrument was close to its sensitivity limits. Thus we obtained spectra and photometry of this unique object for several years to derive an accurate spectral type and the foreground extinction. This allowed us to determine more precisely the distance of the target for the modelling of the dust shell found on Infrared Astronomical Satellite (IRAS) images. Zijlstra \& Weinberger (2002) outline such shells for various types of objects at late stages of their evolution. Their focus was especially on a swept up shell with a void in the interstellar matter (ISM) around the target. This is crucial for both, the "Swiss cheese" like structure of the ISM and for the hydrodynamic evolution of the planetary nebula (PN) built after this evolutionary stage.

We thus investigated the spectroscopic and photometric properties of the central star to provide new input for the complete model of this unique object and its surroundings.

Send offprint requests to: S. Kimeswenger, e-mail: stefan.kimeswenger@uibk.ac.at

* Based on observations at the Innsbruck 60-cm telescope and the Flemish Mercator telescope on Roque de los Muchachos, Spain.

\section{Data}

The spectroscopic data were obtained with the Innsbruck 60-cm telescope (Kimeswenger 2001; Bacher et al. 2001) and an OptoMechanics 10C spectrograph. A CompuScope Kodak 0400 CCD camera was attached to the spectrograph. In the year 2000 a grating with $600 \mathrm{l} / \mathrm{mm}$ was used, resulting in a resolution of $1 \AA$ /pixel. Later a grating with $240 \mathrm{l} / \mathrm{mm}$ (2.6 $\AA$ /pixel) was mounted to achieve a better $\mathrm{S} / \mathrm{N}$ ratio. The complete log of the observations is given in Table 1 . The exposure times varied with the wavelength region from 300 to $900 \mathrm{~s}$. Each spectrum is a composite of individual takes covering about $800 \AA$ with the high resolution grating and $2000 \AA$ with the medium resolution mode. The wavelength shifts were selected to obtain appropriate overlaps of at least $25 \%$ of the wavelength range. In most of the nights several spectra were obtained to improve the $\mathrm{S} / \mathrm{N}$ and eliminate cosmic ray events. The positions along the slit and the starting wavelengths varied to eliminate possible systematic errors in the setup. Flatfield, bias subtraction and wavelength calibration were carried out in a standard manner with the help of MIDAS routines. For comparison the late type standard stars HD 23475 (M 2IIb), HD 39801 (M2I), HD 13325(M3III), HD 40239 (M3II), HD 44478 (M 3III), HD 42995 (M 3III) HD 5316 (M 4III) and HD 12292 (M 5III) were observed with the medium resolution setup. The spectra were not calibrated absolutely, but only corrected for exposure time and airmass (with the atmospheric extinction given by Tüg 1980). 
Table 1. Log of the Innsbruck 60-cm observations.

\begin{tabular}{ccccc}
\hline \hline Date & Mode & Band / & \multicolumn{2}{c}{ Resolution } \\
& & Sampling & Eff.* \\
wavelength & $\AA$ & $\AA$ pixel & $\AA$ \\
\hline 05.02 .2000 & spectra & $6000-9000 \AA$ & 1.0 & 6.2 \\
15.01 .2001 & spectra & $5000-10000 \AA$ & 2.6 & 10.6 \\
18.01 .2001 & spectra & $5000-10000 \AA$ & 2.6 & 10.6 \\
11.02 .2001 & spectra & $5440-10000 \AA$ & 2.6 & 9.9 \\
15.02 .2001 & spectra & $5000-8860 \AA$ & 2.6 & 8.6 \\
16.02 .2001 & spectra & $5440-8860 \AA$ & 2.6 & 8.0 \\
06.03 .2001 & spectra & $5440-8860 \AA$ & 2.6 & 10.7 \\
08.01 .2002 & spectra & $5600-10000 \AA$ & 2.6 & 13.4 \\
09.01 .2002 & spectra & $5000-9000 \AA$ & 2.6 & 14.1 \\
10.12 .2002 & imaging & $B V R I_{\mathrm{C}}$ & & \\
11.12 .2002 & imaging & $B V R I_{\mathrm{C}}$ & & \\
17.01 .2003 & spectra & $5000-10000 \AA$ & 2.6 & 17.1 \\
\hline
\end{tabular}

* Effective resolution was measured as FWHM of night sky lines.

The wide band direct imaging in Innsbruck was obtained with an AP7p SITe 502e CCD device (Kimeswenger et al. 2002a; Lederle \& Kimeswenger 2003). 24 images were taken in the nights of 10th and 11th of December 2002 with $B, V$, $R$ and $I_{\mathrm{C}}$ filters. The exposure times were 30, 20, 10 and $10 \mathrm{~s}$ in $B, V, R$ and $I_{\mathrm{C}}$ respectively. After standard basic CCD reduction the source extraction was performed using SExtractor V2 (Bertin \& Arnouts 1996). The rms of the comparison standards in the field was $<0$. $01\left(\sigma_{B}=0 . \mathrm{m} 003, \sigma_{V}=0.009, \sigma_{R}=0.007\right.$ and $\left.\sigma_{I}=0.004\right)$. Absolute calibration was obtained in both nights using the standard stars HR 580 and HR 596. The rms variations of the zero-points were less than 0.03 , resulting in an error of the mean zero-point of less than 0.015 .

The long term monitoring of the target was done with the P7 photometer attached to the 1.2-m Mercator telescope located at La Palma, using the 7 filters of the Geneva photometric system (Meylan \& Hauck 1981; Golay 1994). The data was obtained and reduced within the framework of monitoring stars with constant airmass as described in Burki et al. (1995). The typical errors derived by the rms of the standard stars for the nights are below $0 \mathrm{~m} 01$. The results are listed in Table 2.

\section{Results}

\subsection{Spectroscopy}

First the spectra were investigated with respect to variability. We cannot detect spectral variability (except in the region of the telluric water vapor bands longwards to $8000 \AA$ - see Fig. 1). Also individual equivalent widths of metal lines of the IR Ca II triplet and of $\mathrm{Mg}$ lead us to the same conclusion. Thus a composite spectrum was derived for the classification. The classification criteria of Kirkpatrick et al. (1991) were applied in the same way as described in Kimeswenger et al. (2003). This results in a spectral class of M 4-M 5. The scheme of TiO indices (Malyuto et al. 1997) argues for an M4.2III star. Applying the extension by Schmidt-Kaler \& Oestreicher (1998) we obtain an M 4III star with $M_{\text {bol }} \approx-2$. $65\left(M_{V} \approx-0\right.$. 5). The relation

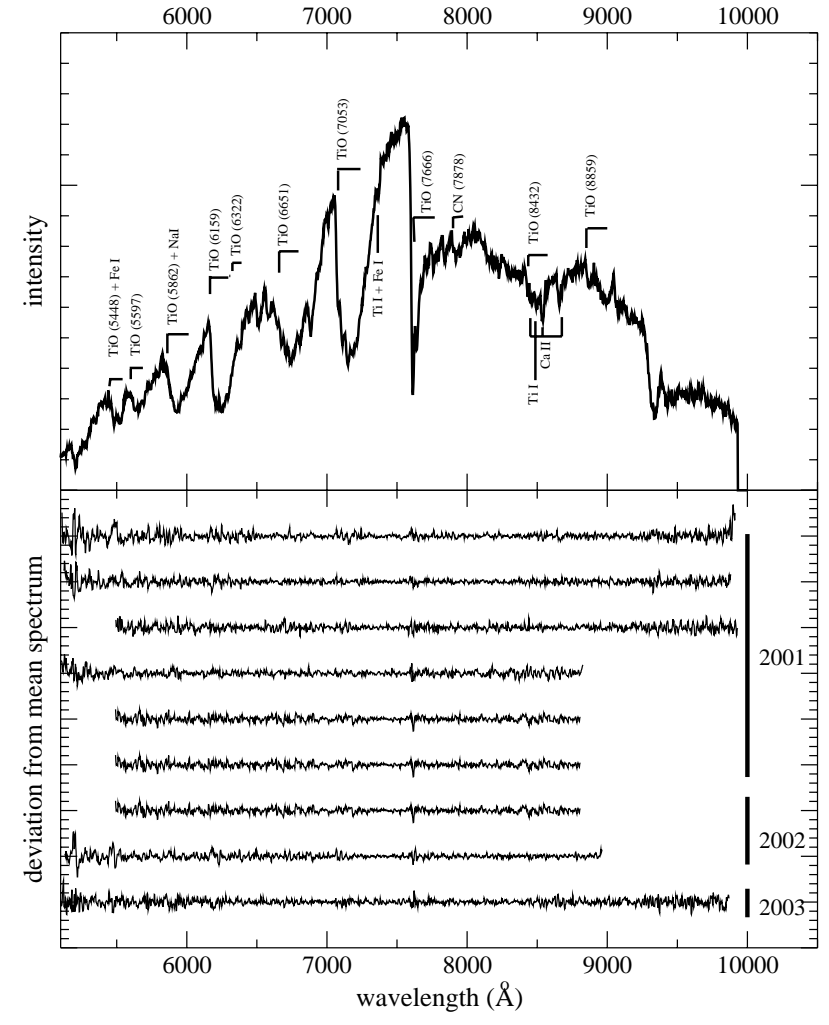

Fig. 1. The average spectrum (upper panel) with the most dominant features identified and the deviation from the mean spectrum of those spectra, using the same instrument setup, during winter 2001, 2002 and 2003. For clarity an offset of 1.0 was added to each spectrum. No spectroscopic variability in the lines or in the continuum trends can be found.

of the $\mathrm{TiO}$ to the $\mathrm{NaD}$ and $\mathrm{Mgb}$ indices propose luminosity class III.

The most reliable classification is the direct comparison with standards, using the identical instrument setup. For this purpose eight comparison stars were taken during February 2001 and January 2003 between the target exposures with the same setup. Especially the TiO features at 7200-7400 $\AA$ and 7700-8000 $\AA$ are extremely sensitive to the effective temperature of the stars in that domain. The latter was not covered by the spectrum used by Zijlstra \& Weinberger (2002). The classification using such bands is completely independent of the correction for the atmospheric and interstellar extinction. The results are shown in Fig. 2. The target superimposes nearly exactly the M4III star HD 5316. The overlay gives us an exact solution for the interstellar extinction of $E_{B-V}=0.43 \pm 0$. 01 .

We tried to use the line ratios of the IR CaII triplet of the comparison stars and those of the target - all taken with the same instrument setup. But not even for the comparison stars, having a much better $\mathrm{S} / \mathrm{N}$ ratio than the target, the effective resolution (see Table 1) allow to derive useful separations between luminosity class III and II.

We favor a spectral type of M4III. The luminosity class remains somewhat uncertain, but is most likely III. This is supported also by the distance (see Sect. 3.2 and discussion in Zijlstra \& Weinberger 2002). 


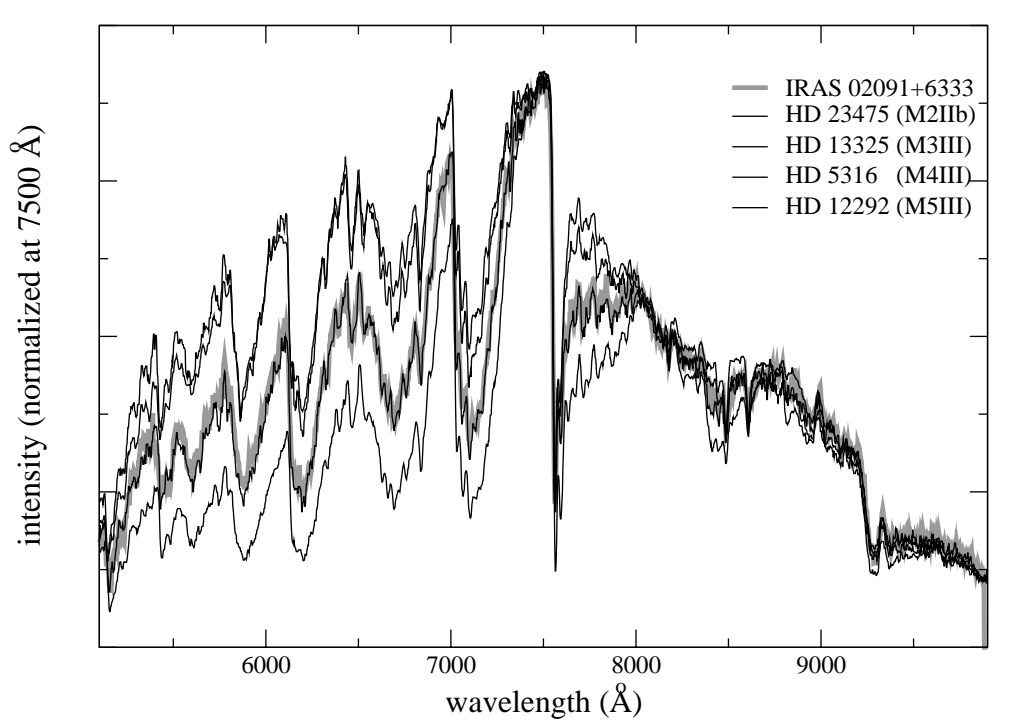

Fig. 2. The composite spectrum of IRAS 02091+6333 (bold line) using an interstellar extinction $E_{B-V}=0.43$ (see text) overlaid with (from top to bottom) the spectra of HD 23475 (M 2IIb), HD 13325 (M 3III), HD 5316 (M 4III) and HD 12292 (M 5III). Those of HD 40239 (M 3II), HD 39801 (M2I), HD 44478 (M 3III) and HD 42995 (M3III) were not plotted for clarity. The spectrum of HD 5316 is almost completely hidden by that of the target.

Table 2. Results of the Mercator photometry in the Geneva system (Meylan \& Hauck 1981).

\begin{tabular}{ccccccccc}
\hline \hline MJD & $v$ & $u$ & $b$ & $b_{1}$ & $b_{2}$ & $v_{1}$ & Weight \\
\hline 52103.63730 & 10.445 & 15.647 & 12.027 & 13.638 & 13.011 & 11.298 & 11.281 & 4 \\
52103.68906 & 10.452 & 15.540 & 12.039 & 13.646 & 12.995 & 11.291 & 11.281 & 4 \\
52104.67404 & 10.450 & 15.643 & 12.017 & 13.615 & 13.007 & 11.287 & 11.286 & 3 \\
52111.70459 & 10.380 & 15.416 & 11.965 & 13.585 & 12.948 & 11.232 & 11.214 & 1 \\
52205.54382 & 10.427 & 15.637 & 12.030 & 13.637 & 13.035 & 11.281 & 11.267 & 2 \\
52299.37930 & 10.421 & 15.427 & 12.045 & 13.700 & 13.010 & 11.280 & 11.261 & 3 \\
52299.42229 & 10.419 & 15.806 & 12.060 & 13.690 & 12.997 & 11.257 & 11.253 & 3 \\
52500.69875 & 10.458 & 15.606 & 12.055 & 13.659 & 13.082 & 11.304 & 11.297 & 2 \\
52501.69090 & 10.453 & 15.472 & 12.047 & 13.676 & 13.067 & 11.306 & 11.286 & 2 \\
52502.69127 & 10.447 & 15.714 & 12.047 & 13.680 & 13.022 & 11.303 & 11.278 & 2 \\
52505.64131 & 10.434 & 15.573 & 12.027 & 13.634 & 13.034 & 11.276 & 11.254 & 3 \\
52506.69780 & 10.421 & 15.552 & 12.026 & 13.650 & 13.018 & 11.279 & 11.258 & 3 \\
52508.69154 & 10.430 & 15.629 & 12.040 & 13.676 & 13.017 & 11.262 & 11.264 & 2 \\
\hline
\end{tabular}

\subsection{Photometry and distance}

We have searched literature and telescope archives for photometry, but found in the optical and in the MIR-FIR only those mentioned already in Zijlstra \& Weinberger (2002) - namely TYCHO in the optical, Midcourse Space Experiment (MSX, Egan et al. 1999) and IRAS in the infrared. The results of our photometry, together with results from literature are collected in Table 3. For the flux to magnitude conversion for IRAS we used the results of Wainscoat et al. (1992) and for MSX that of Cohen et al. (2000). The calibration of IRAS by Hickman et al. (1995) was not taken into account, as the MSX and the IRAS $12 \mu \mathrm{m}$ fluxes gave differences of about 0.40 for all $\mathrm{M}$ type comparison stars here. For the TYCHO measurements we applied the color equations as given in the catalogue: $V=V_{\mathrm{T}}-0.090 \times\left(B_{\mathrm{T}}-V_{\mathrm{T}}\right)$ and $B-V=0.850 \times\left(B_{\mathrm{T}}-V_{\mathrm{T}}\right)$.

During the reviewing process of this work, the 2MASS NIR data was made public. Although we listed the values in Table 3, they were not useful, as the target was overexposed even in the short $53 \mathrm{~ms}$ preexposure of the survey. We thus didn't include these values in our interpretation.

The first TYCHO catalogue (Perryman et al. 1997) and the reanalysis of the same data in TYCHO-2 (Høg et al. 2000) give completely different results for the $B_{\mathrm{T}}$ band. The $V_{\mathrm{T}}$ band differs considerably more than the error given in the catalogue. Thus we assume the error to be significantly higher. For further analysis we use the TYCHO-2 values.

The Mercator monitoring (Fig. 3) clearly shows an irregular variability with an amplitude below 0.1 in $V$.

On the other hand the variation between our measurements, those of the amateur observations reported in Zijlstra \& Weinberger (2002) and TYCHO-2 in the optical give an amplitude of some tenths of a magnitude. The $\left(V_{\mathrm{Tmin}}-V_{\mathrm{Tmax}}\right)$ value (15\% and $85 \%$ of the 228 photometric transits) given in TYCHO-2 is 1 m.71. But we assume the latter is mainly due to the limits of the experiment.

The MSX and IRAS measurements together with the spectroscopic stability lead us to the conclusion that the object seems to be very stable in the effective temperature. That fact limits the optical photometric amplitude to those found in the Mercator monitoring. Such small variations seem to be usual for late type stars around the tip of the first giant branch (Ita et al. 2002).

The colors $(V-R)_{0}=1.15$ and $\left(V-I_{\mathrm{C}}\right)_{0}=2$ m 68 indicate a M 4.5III MK class (Drilling \& Landolt 2000). Using our 
Table 3. Summary of photometric observations except those listed in Table 2. The TYCHO measurements were converted to the standard system (see text).

\begin{tabular}{|c|c|c|c|c|}
\hline date & Band & Mag. & $\begin{array}{l}\text { Quality } \\
\text { or error }\end{array}$ & Ref. \\
\hline \multirow[t]{4}{*}{$10 / 11.12 .2002$} & $B$ & $122^{\mathrm{m}} 607$ & 0.014 & here \\
\hline & V & $10^{\mathrm{m}} \cdot 519$ & 0.009 & here \\
\hline & $R$ & 9.077 & $0^{\mathrm{m}} 004$ & here \\
\hline & $I_{\mathrm{C}}$ & 7. 309 & 0.019 & here \\
\hline \multirow[t]{4}{*}{$\approx 1989$} & $B$ & $12 \mathrm{~m} 22$ & 0.23 & TYC-1 \\
\hline & V & $10^{\mathrm{m}} 41$ & 0.05 & TYC-1 \\
\hline & $B$ & 12.46 & $0^{\mathrm{m}} \cdot 23$ & TYC-2 \\
\hline & $V$ & 10.37 & 0.05 & TYC-2 \\
\hline \multirow[t]{4}{*}{ January 2000} & $B$ & 12.55 & $?$ & [1] \\
\hline & $V$ & $10^{\mathrm{m}} .5$ & $?$ & [1] \\
\hline & $R$ & 9.9 & $?$ & [1] \\
\hline & $I$ & $7 \mathrm{~m} \cdot 4$ & $?$ & [1] \\
\hline \multirow[t]{4}{*}{ January 2001} & $B$ & 11.7 & $?$ & [1] \\
\hline & $V$ & $10^{\mathrm{m}} 1$ & $?$ & [1] \\
\hline & $R$ & 9.3 & $?$ & [1] \\
\hline & $I$ & 7.3 & $?$ & [1] \\
\hline \multirow[t]{3}{*}{5.1 .1999} & $J$ & $5.326^{\#}$ & $0^{\mathrm{m}} 03$ & 2MASS \\
\hline & $H$ & 4. $350^{\#}$ & 0.29 & 2MASS \\
\hline & $K_{\mathrm{s}}$ & 4. $034^{\#}$ & 0.34 & 2MASS \\
\hline \multirow[t]{2}{*}{$\approx 1995$} & [8.3] & 3.80 & qal 4 & MSX \\
\hline & [14.6] & 2.9 & qal 1 & MSX \\
\hline \multirow[t]{2}{*}{$\approx 1983$} & [12] & 3.76 & qal 3 & IRAS \\
\hline & [25] & 3. 55 & qal 3 & IRAS \\
\hline
\end{tabular}

[1] Zijlstra \& Weinberger (2002).

\# The 2MASS data is flagged in the data base as

"radial profile fitting of overexposed source" and "profile fit very poor".

Table 4. Summary of the results for IRAS 02091+6333.

\begin{tabular}{ll}
\hline \hline Spectral type & $\mathrm{M} 4_{ \pm 0.3} \mathrm{III}$ \\
$T_{\text {eff }}$ & $3350_{ \pm 50} \mathrm{~K}$ \\
Type of photometric variability & irr. or SR \\
$<V>_{\text {Johnson }}$ & $10^{\mathrm{m}} 52_{ \pm 0.03}$ \\
Interstellar $E_{B-V}$ & $0.43_{ \pm 0.01}$ \\
Visual amplitude & $\leq 0.1$ \\
$M_{V}$ & -0.5 \\
$M_{\text {bol }}$ & -2.65 \\
Distance & $1020_{-150}^{+130} \mathrm{pc}$ \\
Luminosity & $920_{ \pm 150} L_{\odot}$ \\
\hline
\end{tabular}

photometry and the color equations above we receive $V_{\mathrm{T}}-$ $[8.3]=5.56$. Cohen et al. (2000) are announcing 5.21, 5.58 and 6.02 for M 3, M 4 and M 5 respectively, which is also consistent with the classifications found above. The Mercator monitoring was not used for the colors, as this system is lacking investigations, absolute calibrations and color equations for very late type stars (Meylan \& Hauck 1981; Moro \& Munari 2000).

We derived, again using TYCHO, MSX measurements and HIPPARCOS parallaxes, the spectral energy distributions (SED) for M3-5 giants from our spectroscopic sample and from those one of van Belle et al. (1999). The latter is a sample of stars investigated in detail with interferometers for the determination of accurate size and luminosity of late type stars.

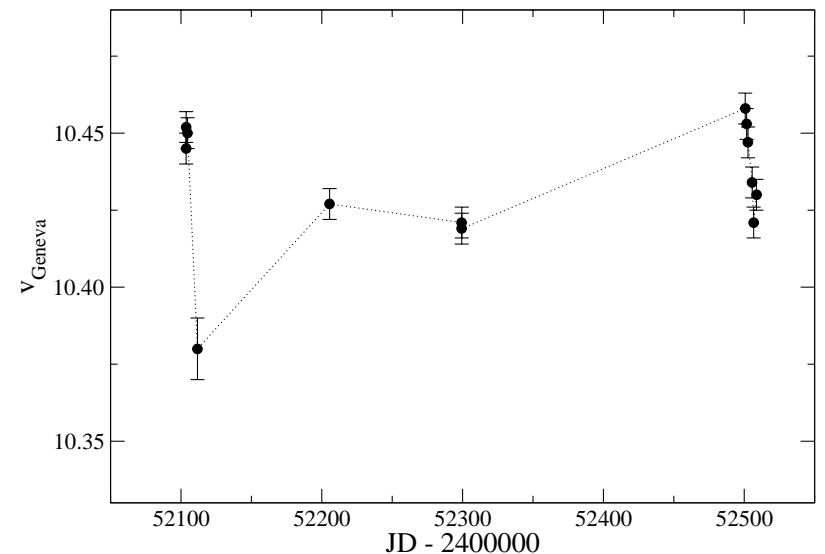

Fig. 3. The Mercator photometry shows a weak photometric variation on medium to long timescales.

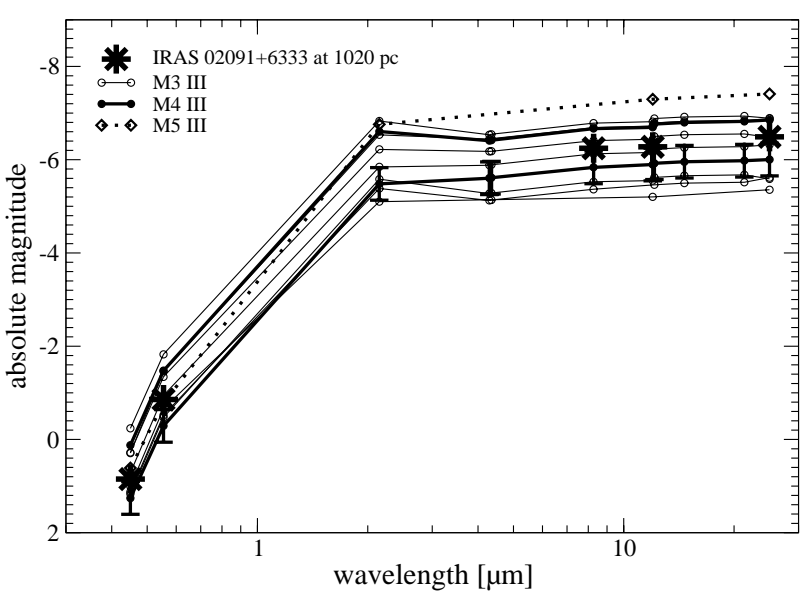

Fig. 4. The SEDs of the M 3-5 III stars of the sample from van Belle et al. (1999) and of the stars used for spectral comparison here. The distances for calibration of the absolute magnitude were taken from HIPPARCOS. The photometry was taken from $\operatorname{SIMBAD}(B, V)$, IRC $(K$, Neugebauer \& Leighton 1969), MSX $(4-21 \mu \mathrm{m})$ and IRAS (12 and $25 \mu \mathrm{m}$ ). The error bars give the tolerance added by the HIPPARCOS errors for the worst case star. The target fits well within the two M4III stars assuming a distance of $1020 \mathrm{pc}$.

Two stars - namely HD 13325 (M 3III) and HD 5316 (M 4III) overlap between their and our sample. One can clearly see in Fig. 4 the broad band mid-IR flux hardly varies between M3 and M 4 stars. Only M 5 stars have a significant different SED. We thus fit our target between the M 4 stars of the sample. This provides us with (using all bands) a distance of $1020 \mathrm{pc}$. The HIPPARCOS errors for the bright comparison stars and the uncertainties from the photometry of the target results in a lower limit of $870 \mathrm{pc}$ and an upper one of $1150 \mathrm{pc}$. The luminosity derived from the spectroscopy and the $V$ photometry give us $920 \mathrm{pc}$. According to van Belle et al. (1999) for $K-[12]>0.6$ there is no correlation with the effective temperature anymore. Thus this band, lacking here, would deliver us no additional information. 


\subsection{Astrometry}

The target was suspected to have a relatively high proper motion in the TYCHO catalogue, although the positional error given there is very high. We thus tried to derive a value for the proper motion in the same way as in Kimeswenger et al. (2002b) by using the six sky survey plates of the region. But as the target is extremely overexposed and lies near the corner of the POSS-E (1952 and 1993) and of the Quick-VN (1983) plates, we cannot derive a proper solution. The accuracy is about the same as in the the TYCHO experiment. Kislyuk et al. (1999) give similar numbers in their online version, using their plate from 1983 and the AC2000 catalogue position from 1905 (Urban et al. 1997). But forwarding the errors in both catalogues to the resulting proper motion we again end up with large error bars $\left(12 \pm 7\right.$ mas $\left.\mathrm{yr}^{-1}\right)$. The direction of the motion vector is co-lined with the TYCHO result, suggesting a genuine movement which leads to a motion of about $60 \mathrm{~km} \mathrm{~s}^{-1}$ relative to the surrounding stellar field after subtracting the galactic ro-

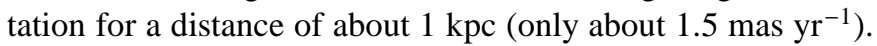
This is, although still possible, very high for galactic disk stars. The vector is almost opposite to the galactic rotation parallel to the galactic plane. Further investigations about the population membership are needed.

\section{Conclusions}

The precise spectroscopic classification of the central star IRAS $02091+6333$ of the unique giant dust shell reported in Zijlstra \& Weinberger (2002) allowed us to derive a more accurate distance of the target. This leads us to a somewhat higher mass estimate of $5.2 M_{\odot}$ and a size of $1.8 \times 10^{17} \mathrm{~m}(5.2 \mathrm{pc})$ and thus even enlarges the discrepancy in lifetime as discussed in Sect. 3.3 of Zijlstra \& Weinberger (2002) and therefore clearly excludes the scenario of the mass originating mainly from the star itself. This result is strengthened by the fact of the lower luminosity we derived (40\% below the one estimated there). The star lies in the evolutionary tracks (Girardi et al. 2000a) nearby the red giant branch tip and rather far from the TP-AGB. The small irregular photometric variability fits well to this assumption (Ita et al. 2002). So the model of the swept up ISM is strongly supported by our measurements. The fast movement, although uncertain, may shorten the timescales for the bubble. This may suggest a wind having a higher momentum. One can only speculate on the origin of this additional source of energy. There is no signature in the spectroscopy for a hot companion causing a symbiotic Mira. Also Schmeja \& Kimeswenger (2001) have shown that those objects have clear signatures in their NIR and MIR bands.

IRAS $02091+6333$ has a distance of $D=1020_{-150}^{+130} \mathrm{pc}$, a luminosity of $L=920 \pm 150 L_{\odot}$ and, according to van Belle et al. (1999), an effective temperature of $T_{\text {eff }}=3350 \pm 50 \mathrm{~K}$. Both the spectroscopy as well as the photometric consistency of the colors with the MK type from visual to IR bands suggest there is currently no dominant circumstellar shell material. Girardi et al. (2000b) discussed inhomogeneities in the tracks and in the evolution due to different He-core burning phases. It affects slightly the luminosity (and thus the distance) but hardly the photometric colors (Girardi et al. 2000a). This adds some uncertainties to the values derived for individual sources.

Acknowledgements. We thank the colleagues of the institute for the unlimited access to the facilities of the new university observatory in Innsbruck. The staff of the "Instituut voor Sterrenkunde", KULeuven is acknowledged for the photometric monitoring at the Mercator facilities and the staff of the Geneva observatory is acknowledged for the photometric reduction

\section{References}

Bacher, A., Lederle, C., Grömer, G., et al. 2001, IBVS, 5182 van Belle, G. T., Lane, B. F., Thompson, R. R., et al. 1999, AJ, 117, 521

Bertin, E., \& Arnouts, S. 1996, A\&AS, 117, 393

Burki, G., Rufener, F., Burnet, M., et al. 1995, A\&AS, 112, 383

Cohen, M., Hammersley, P. L., \& Egan, M. P. 2000, AJ, 120, 3362

Drilling, J. S., \& Landolt, A. U. 2000, in Allen's astrophysical quantities, 4th ed. (New York: AIP Press; Springer), ed. A. N. Cox, 381

Egan, M. P., Price, S. D., Moshir, M. M., et al. 1999, The Midcourse Space Experiment Point Source Catalog, Version 1.2, Air Force Research Lab. Technical Rep. AFRL-VS-TR-1999-1522

Girardi, L., Bressan, A., Bertelli, G., \& Chiosi, C. 2000a, A\&AS, 141, 371

Girardi, L., Mermilliod, J.-C., \& Carraro, G. 2000b, A\&A, 354, 892

Golay, M. 1994, in The MK process at 50 years, Proceedings of a Workshop of the Vatican Observatory, held in Tucson Arizona, USA (San Francisco: Astronomical Society of the Pacific, ASP), ed. C. Corbally, R. O. Gray, \& R. F. Garrison, 164

Hickman, M. A., Sloan, G. C., \& Canterna, R. 1995, AJ, 110, 2910

Høg, E., Fabricius, C., Makarov, V. V., et al. 2000, A\&A, 355, L27

Ita, Y., Tanabé, T., Matsunaga, N., et al. 2002, MNRAS, 337, L31

Kislyuk, V., Yatsenko, A., Ivanov, G., Pakuliak, L., \& Sergeeva, T. 1999, The FON Astrographic Catalogue, Main Astronomical Observatory of National Academy of Science of Ukraine

Kimeswenger, S. 2001, AGM, 18, 251

Kimeswenger, S., Lederle, C., Schmeja, S., \& Armsdorfer, B. 2002a, MNRAS, 336, L43

Kimeswenger, S., Schmeja, S., Kitzbichler, M. G., et al. 2002b, IBVS, 5233

Kimeswenger, S., Lederle, C., Armsdorfer, B., \& Pritchard, J. 2003, Rev. Mex. Astron. Astrofis., 39, 33

Kirkpatrick, J. D., Henry, T. J., \& McCarthy Jr., D. W. 1991, ApJS, 77,417

Lederle, C., \& Kimeswenger, S. 2003, A\&A, 397, 951

Malyuto, V., Oestreicher, M. O., \& Schmidt-Kaler, Th. 1997, MNRAS, 286, 500

Meylan, G., \& Hauck, B. 1981, A\&AS, 46, 281

Moro, D., \& Munari, U. 2000, A\&AS, 147, 361

Neugebauer, G., \& Leighton, R. B. 1969, NASA SP, Washington: NASA

Perryman, M. A. C., \& ESA 1997, The Hipparcos and Tycho catalogues. Astrometric and photometric star catalogues derived from the ESA Hipparcos Space Astrometry Mission (Noordwijk, Netherlands: ESA Publications Division), ESA SP-1200

Schmeja, S., \& Kimeswenger, S. 2001, A\&A, 377, L18

Schmidt-Kaler, Th., \& Oestreicher, M. O. 1998, AN, 319, 375

Tüg, H. 1980, A\&A, 82, 195

Wainscoat, R. J., Cohen, M., Volk, K., Walker, H. J., \& Schwartz, D. E. 1992, ApJS, 83, 111

Urban, S. E., Corbin, T. E., \& Wycoff, G. L. 1997, The ACT Reference Catalog, U.S. Naval Observatory, Washington D.C.

Zijlstra, A., \& Weinberger, R. 2002, ApJ, 572, 1006 